\title{
Elementos que integram listas de verificação para cirurgia cardíaca segura
}

\author{
Elements that integrate checklists for safe cardiac surgery \\ Elementos que integran listas de verificación para una cirugía cardíaca segura
}

Recebido: 15/12/2021 | Revisado: 22/12/2021 | Aceito: 04/01/2022 | Publicado: 05/01/2022

\author{
Alba Regina Cartaxo Sampaio Thomé \\ ORCID: https://orcid.org/0000-0002-5385-1357 \\ Universidade Federal de Alagoas, Brasil \\ E-mail: regina.cartaxo@outlook.com \\ Thaís Honório Lins Bernardo \\ ORCID: https://orcid.org/0000-0002-8058-8400 \\ Universidade Federal de Alagoas, Brasil \\ E-mail: thais.bernardo@eenf.ufal.br
}

\begin{abstract}
Resumo
Objetivo: buscar evidências disponíveis na literatura sobre elementos que integram listas de verificação para cirurgia cardíaca segura. Metodologia: trata-se de uma revisão integrativa, realizada entre setembro e outubro de 2020, estudos nos idiomas inglês, português e espanhol com abordagem qualitativa e quantitativa que respondessem à pergunta norteadora. Não foi empregado recorte temporal em razão do número limitado de artigos que responderam aos critérios de inclusão. Este artigo originou-se do projeto de pesquisa intitulado "Construção e validação de instrumento para a assistência em cirurgia cardíaca segura" aprovado Comitê de Ética em Pesquisa da Universidade Federal de Alagoas, e com base no parecer emitido pelo número 15410219.6.0000.5013. Resultados: os artigos incluídos conforme os critérios de elegibilidade para a revisão integrativa apresentaram elementos de segurança voltados a especificidade cirúrgica, oito artigos foram encontrados e pode-se perceber itens apropriados e direcionados a segurança cirúrgica do paciente cardíaco. Conclusão: os itens específicos para cirurgia cardíaca foram diversos, reunindo informações claras e objetivas quanto a segurança para o paciente cardíaco cirúrgico.
\end{abstract}

Palavras-chave: Lista de checagem; Cirurgia torácica; Procedimentos cirúrgicos torácicos; Segurança do paciente; Cirurgia geral.

\begin{abstract}
Objective: to seek evidence available in the literature on elements that make up checklists for safe cardiac surgery. Methodology: this is an integrative review, carried out between September and October 2020, studies in English, Portuguese and Spanish with a qualitative and quantitative approach that answered the guiding question. Time frame was not used due to the limited number of articles that met the inclusion criteria. This article originated from the research project entitled "Construction and validation of an instrument for care in safe cardiac surgery", approved by the Research Ethics Committee of the Federal University of Alagoas, and based on the opinion issued under number 15410219.6.0000.5013. Results: the articles included in accordance with the eligibility criteria for the integrative review presented safety elements aimed at surgical specificity, eight articles were found and it was possible to see appropriate items aimed at the surgical safety of cardiac patients. Conclusion: specific items for cardiac surgery were diverse, bringing together clear and objective information regarding safety for cardiac surgical patients.
\end{abstract}

Keyword: Checklist; Thoracic surgery; Thoracic surgical procedures; Patient safety; General surgery.

\section{Resumen}

Objetivo: buscar la evidencia disponible en la literatura sobre los elementos que componen las listas de verificación para una cirugía cardíaca segura. Metodología: se trata de una revisión integradora, realizada entre septiembre y octubre de 2020, estudios en inglés, portugués y español con un enfoque cualitativo y cuantitativo que respondió a la pregunta orientadora. No se utilizó el marco de tiempo debido al número limitado de artículos que cumplieron con los criterios de inclusión. Este artículo tiene su origen en el proyecto de investigación titulado "Construcción y validación de un instrumento para la atención en cirugía cardíaca segura", aprobado por el Comité de Ética en Investigación de la Universidad Federal de Alagoas, y en base al dictamen emitido con el número 15410219.6.0000.5013. Resultados: los artículos incluidos de acuerdo con los criterios de elegibilidad para la revisión integrativa presentaron elementos de seguridad orientados a la especificidad quirúrgica, se encontraron ocho artículos y fue posible ver los ítems adecuados dirigidos a la seguridad quirúrgica de los pacientes cardíacos. Conclusión: los ítems específicos para cirugía cardíaca fueron diversos, reuniendo información clara y objetiva sobre la seguridad del paciente quirúrgico cardíaco.

Palabras clave: Lista de verificación; Cirugía torácica; Procedimientos quirúrgicos torácicos; Seguridad del paciente; Cirugía general. 


\section{Introdução}

Estima-se que 134 milhões de eventos adversos ocorrem a cada ano devido ao atendimento inseguro em hospitais de países com baixa e média renda, contribuindo para 2,6 milhões de mortes anualmente (World Health Organization, 2019). Um milhão de mortes a cada ano ocorrem devido a complicações pós cirúrgicas, sendo 50\% evitáveis (Weiser \& Gawande, 2015).

Dentre as cirurgias, a cardiovascular é considerada uma das mais complexas e pode se beneficiar ao administrar parte dessa complexidade por meio do uso de listas de verificação (Konfirst et al., 2015). O uso de checklist cirúrgico é uma estratégia para promoção da melhoria do cuidado, reduzindo complicações e evento adverso, é de baixo custo e promissor para realidades com recursos limitados (Vivekanantham et al., 2014; Russ et al., 2015).

A Organização Mundial da Saúde (OMS) lançou uma lista de verificação de segurança que deve ser adaptada ao sistema atual (World Health Organization, 2011). As diretrizes e lista de verificação de segurança cirúrgica publicada pela OMS é genérica e não especificamente adaptadas para resolver os problemas do paciente e fatores de risco de subespecialidades cirúrgicas. Diferentes subespecialidades devem desenvolver formatos específicos para proporcionar segurança ao paciente cirúrgico (Kim et al., 2015).

A implementação completa de listas de verificação de segurança cirúrgica tem sido associada a melhores resultados, com adições e modificações na prática local do serviço (Organização Mundial da Saúde, 2009). A prática diária reafirma que embora os instrumentos já utilizados incluam fatores gerais de risco, muitas vezes não incluem fatores específicos para as cirurgias cardíacas como: utilização de circulação extracorpórea, tempo de coagulação ativado, quantidade de heparina, preparação pré operatória, temperatura do paciente a ser atingida, quando comparados à lista primitiva (Thomé et al., 2017). E além de ser útil para a segurança do paciente, o checklist contribui para gerenciar dados de forma segura e gerar a construção de indicadores hospitalares (Bernardo et al., 2021).

Deste modo, o artigo objetivou buscar evidências disponíveis na literatura sobre elementos que integram listas de verificação para cirurgia cardíaca segura, partindo da seguinte pergunta de pesquisa: Quais elementos integram as listas de verificações para cirurgia cardíaca segura?

\section{Metodologia}

A busca dos artigos da revisão integrativa foi realizada entre setembro e outubro de 2020, nas bases de dados Medline via Pubmed (Nacional Library of Medicine National Institutes of Health) por meio de descritores em inglês Mesh (Medical Subject Headings), Cinhal (Cumulative Index to Nursing and Allied Health Literature) nos títulos (Headings), Lilacs (Literatura Latino Americana e do Caribe em Ciências da Saúde) pelo (Descs), Scopus e Web of Scienc com palavras chaves.

A revisão integrativa (RI) foi composta por seis etapas: definição da pergunta de pesquisa, busca e seleção dos estudos primários, extração de dados dos estudos primários, avaliação crítica dos estudos primários, síntese dos resultados da revisão, apresentação da revisão (Mendes et al., 2019). Para a compreensão do conteúdo abordado foi elaborado as estratégias de busca dos estudos primários e foram combinadas conforme cada elemento da estratégia PICo (P - População/Paciente/problema; I Interesse; Co- Contexto) (Joanna Briggs Institute, 2014). O (P) Segurança do paciente (I) checklists (Co) cirurgia cardíaca.

$\mathrm{Na}$ inclusão dos artigos foi adotado os seguintes critérios: estudos nos idiomas inglês, português e espanhol; artigos com abordagem qualitativa e quantitativa que respondessem à pergunta norteadora. Foram excluídos os artigos duplicados, teses, dissertações e capítulos de livros. Não foi empregado recorte temporal em razão do número limitado de artigos que responderam aos critérios de inclusão.

Procedeu-se a análise inicialmente pela leitura do título e do resumo das produções, com o intuito de verificar a adequação aos critérios estabelecidos, no segundo momento, houve a leitura na integra das publicações selecionadas. Dois 
revisores independentes realizaram a pesquisa, no caso de discordância entre os revisores, um terceiro revisor foi consultado e chegado a um consenso.

Este artigo originou-se do projeto de pesquisa intitulado "Construção e validação de instrumento para a assistência em cirurgia cardíaca segura” aprovado Comitê de Ética em Pesquisa da Universidade Federal de Alagoas, e com base no parecer emitido pelo número 15410219.6.0000.5013.

\section{Resultados}

Os artigos incluídos conforme os critérios de elegibilidade para a revisão integrativa apresentaram elementos de segurança voltados a especificidade cirúrgica, oito artigos foram encontrados conforme Figura 1.

Figura 1- Fluxograma das etapas de seleção duplo-independente (n=08). Maceió, AL, Brasil, 2020.

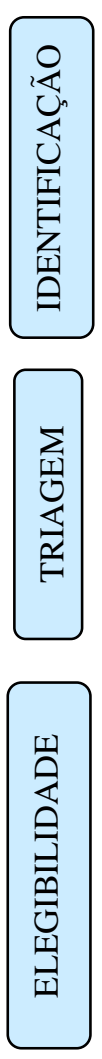

\section{Registros identificados por meio de pesquisa de banco de dados

$$
(n=284)
$$

\section{Registros adicionais identificados por} outras fontes

$$
(\mathrm{n}=0)
$$
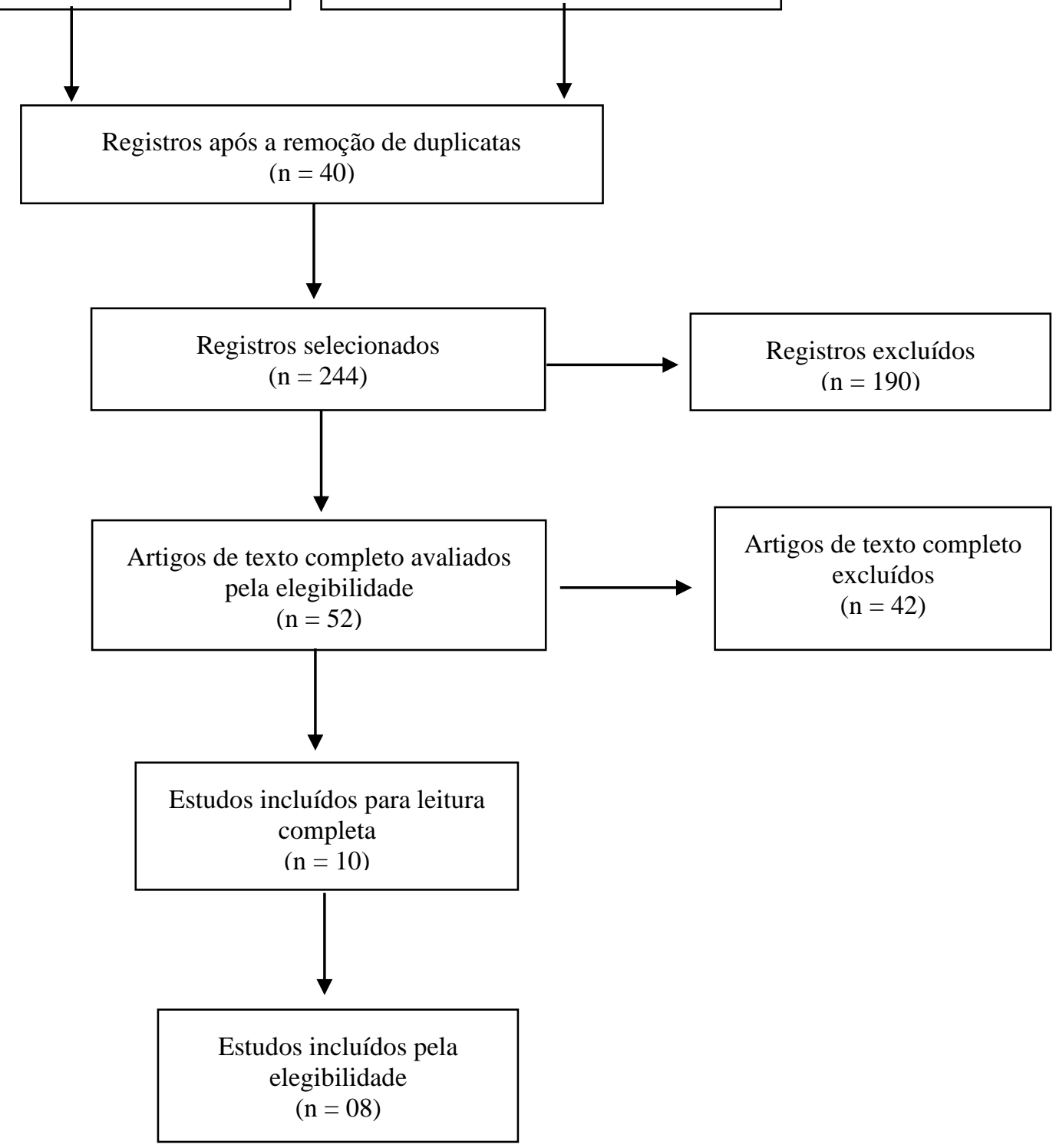

Fonte: Dados da pesquisa (2020). 
Nestes artigos pode-se perceber itens apropriados e direcionados a segurança cirúrgica do paciente cardíaco, a Tabela 1 abaixo descreve a os artigos selecionados.

Tabela 1 - Artigos selecionados conforme título, país, autor e ano do artigo. Maceió, Al, Brasil, 2020.

\begin{tabular}{|c|c|}
\hline Título & País/ Autor/Ano \\
\hline $\begin{array}{l}\text { Surgical time out checklist with debriefing and multidisciplinary feedback improves } \\
\text { venous thromboembolism prophylaxis in thoracic surgery: a prospective audit. }\end{array}$ & $\begin{array}{l}\text { United Kingdom (UK) } \\
\text { Berrisford et al., (2011) }\end{array}$ \\
\hline Lessons from aviation - the role of checklists in minimally invasive cardiac surgery. & $\begin{array}{l}\text { Canadá } \\
\text { Hussain et al., (2015) }\end{array}$ \\
\hline Checklists and Safety in Pediatric Cardiac Surgery. & $\begin{array}{l}\text { United States of América } \\
\text { (USA) } \\
\text { Konfirst et al., (2015) }\end{array}$ \\
\hline $\begin{array}{l}\text { Developing a Cardiopulmonay Bypass Separation Checklist:Consensus Via a } \\
\text { Modified Delphi Technique. }\end{array}$ & $\begin{array}{l}\text { Texas, United States of América } \\
\text { (USA) } \\
\text { Ogden et al., (2016) }\end{array}$ \\
\hline $\begin{array}{l}\text { Improved Compliance and Comprehension of a Surgical Safety Checklist With } \\
\text { Customized Versus Standar Training: A Randomized Trial. }\end{array}$ & $\begin{array}{l}\text { Índia } \\
\text { Rakoff et al., (2015) }\end{array}$ \\
\hline When a checklist is not enough: How to improve them and what else is needed. & $\begin{array}{l}\text { Boston, Mass, United States of } \\
\text { América (USA) } \\
\text { Raman et al., (2016) }\end{array}$ \\
\hline The use of checklist as a method to reduce human error on cardiac operating rooms. & $\begin{array}{l}\text { Richmond, Virginia, United } \\
\text { States of América (USA) } \\
\text { Spiess B. D. (2013) }\end{array}$ \\
\hline $\begin{array}{l}\text { Briefing and debriefing in the cardiac operating room. Analysis of impact on theatre } \\
\text { team attitude and patient safety. }\end{array}$ & $\begin{array}{l}\text { United Kingdom (UK) } \\
\text { Papaspyros et al., (2010) }\end{array}$ \\
\hline
\end{tabular}

Fonte: Dados da pesquisa (2020).

$\mathrm{O}$ artigo (Berrisford et al., 2012) relaciona a pergunta do anestesista e a resposta do cirurgião na fase Time out com ênfase em nove perguntas com o paciente já anestesiado, dentre estas perguntas observa-se algumas que coincidem com perguntas gerais de segurança do checklist padrão da OMS e outras que se direcionam a riscos específicos como: A heparina foi administrada?"

O artigo (Hussain et al., 2016) apresenta 22 checagens sequenciadas, uma lista de verificação que descreve as etapas de inserção de cânulas para procedimentos minimamente invasivos em cirurgia cardíaca, confirmações direcionadas como: unidades de heparina por litro no sistema de circulação extracorpórea, controle do tempo de coagulação ativada, heparina administrada sistemicamente no paciente, inserção de cânula femoral arterial e venosa para cirurgia minimamente invasiva.

O artigo (Konfirst et al., 2015) descreve uma lista de verificação desenvolvida para um programa cardíaco pediátrico com direcionamento de checagens para os profissionais, na fase Sign in checagens como: nome, peso, altura, tamanho da válvula do paciente e disponibilidade de uso do ecocardiograma. No Time out é verificado posição do paciente para minimizar o risco do decúbito, testagem do desfibrilador, lado de realização da toracotomia, necessidade de produtos sanguíneos específicos como: plaquetas, crioprecipitado, plasma fresco congelado, necessidade de gás especial como: óxido nítrico, CO2, nitrogênio, confirmação de checagem de cânulas pelo cirurgião. No Sign out confirmação de necessidade de ECMO (membrana de oxigenação extracorpórea).

No artigo de (Ogden et al., 2016) é observado uma lista de verificação a ser utilizada no período de separação da circulação extracorpórea para decisões, avaliações e pontos de comunicação entre cirurgião, anestesista e perfusionista. Checagens como temperatura acima de $36^{\circ} \mathrm{C}$, retorno do ritmo cardíaco normal, necessidade de marcapasso externo, remoção 
de ar do coração e dos enxertos, necessidade de uso de inotrópicos e vasopressores são confirmações direcionadas a cirurgia cardíaca.

Em (Rakoff et al., 2018) foram observadas confirmações prévias do preparo da pele do paciente, se pré medicações foram prescritas, disponibilidade de cânulas e tamanhos, necessidade perfusão seletiva cerebral, colocação de gelo na cabeça, tipo de prime utilizado na circulação extracorpórea, checagem do sensor de ar da circulação extracorpórea, se houve quebra da técnica cirúrgica. O artigo de (Raman et al., 2016) descreve uma lista de verificação pré-operatória com perguntas sobre anticoagulação profilática e precaução do uso de medicações.

Em (Spiess 2013) um checklist é descrito em duas tabelas, na tabela um confirmações a serem utilizadas pela perfusão acerca da identificação do paciente, esterilização dos componentes a serem utilizados, máquina coração pulmão, resfriamento do coração, suprimento de gás, bomba, condição da tubulação da bomba inspecionada, checagem da monitorização, segurança e alarmes, oxigenador, tubulação e acessórios. Na tabela dois descreve checagens de cada profissional com inclusão de debriefing ao final do checklist, aborda conferência da máquina coração pulmão, resfriamento do coração e suplementação de gás anestésico durante a bypass.

Em (Papaspyros et al., 2010) o controle de glicemia, necessidade de indicação de beta bloqueador e aplicação de segunda dose do antibiótico profilático foram apontadas. Trata-se de um checklist com repostas dicotômicas dividido em processo de etapa geral, briefing com onze itens e debriefing com seis itens.

\section{Discussão}

De modo geral os artigos analisados permitiram identificar confirmações de uso rotineiro em cirurgias cardíacas e juntas estas verificações podem melhorar a comunicação por meio de modelos mentais compartilhados. Em situações de rotina ou emergência checklist são altamente eficaz e representam um esforço coordenado da equipe, exigindo que várias pessoas capacitadas se comuniquem e tomem as devidas providências (Spiess 2013; Chan et al., 2019).

Muitos itens analisados nos artigos da revisão integrativa reafirmaram os itens básicos de verificação encontrados na lista tradicional o que nos faz compreender que apesar dos artigos abordarem confirmações especificas e direcionadas de segurança para a cirurgia cardíaca mantém o padrão e a estrutura orientado pela OMS.

Um estudo realizado na China foi evidenciado que o checklist sugerido pela OMS continua sendo uma ferramenta poderosa para a segurança do paciente cirúrgico e que listas de verificações são intervenções comportamentais, o que significa que exigem uma mudança no comportamento da equipe da sala de operação para ser eficaz (Tan et al., 2021).

Abaixo na Tabela 2, é descrito os elementos que integram listas de verificação em cirurgia cardíaca e suas respectivas fases de confirmação encontrada nos artigos. 
Tabela 2 - Elementos que integram listas de verificação para cirurgia cardíaca e fase cirúrgica. Maceió, Al, Brasil, 2020.

Elementos
Is glycaemic control/beta blockers indicated?
Is the patient positioned to minimize injury?

Transplant Only: Donor blood type compatible with recipient?

Transplant Only: Anti Rejection Drugs drawn up and ready?

Special gases needed? (nitric oxide, $\mathrm{CO2}$, nitrogen, if so call respiratory)

Are cannulas on the field and checked by surgeon?

Patient temperature is $>36^{\circ}$ Celsius

Patient risk level (Low/Med/High)

Are perfusion and nurses ready?

Has perfusion checklist been performed?

Cannulae checked and available?

Cannulae size

Bypass prime (blood vs. clear)

Tardetes core temp

Is air sensor put on?

Use or non-use DHCA, selective cerebral perfusion?

Icepack on head?

Other bypass consideration (shunt collaterals, LV vent, cardioplegia etc.)

Breaks in technique

ATC control sample to perfusionists

$\mathbf{5 , 0 0 0}$ units heparin given (systemically) to patient by anesthesia

Patient systemically heparinized
Fase cirúrgica

Briefing

Briefing

Time out

Time out

Time out

Time out

Time out

Before Anesthesia Begins

Before Anesthesia Begins

Before Skin Incision

Before Skin Incision

Before Skin Incision

Before Skin Incision

Before Skin Incision

Before Skin Incision

Before Skin Incision

Before Skin Incision

Before Skin Incision

Before Sternal Closure

Time out

Time out

Time out

Fonte: Dados da pesquisa (2020).

Ao analisar as fases cirúrgicas e os itens de verificação observa-se que a maior parte das checagens se concentram antes ou durante o Time Out (antes da incisão de pele), visto que são as fases que requerem a confirmação da maioria das etapas antes de prosseguir com o início da cirurgia, fator de grande impacto para minimizar a ocorrência de eventos adversos.

Há também consenso entre os estudos que os instrumentos são complementares dentro do processo de trabalho multiprofissional. É necessário gestão de recursos humanos, materiais, comunicação, habilidades técnicas e treinamento entre os profissionais para utilização dos recursos para segurança do paciente. E o conhecimento de como implementar intervenções evidenciadas de forma eficaz e sustentável em escala permanente faz parte da segurança do paciente (White et al., 2021).

Recomenda-se a utilização dos checklist deste estudo para a segurança do paciente em cirurgia cardíaca como modelo para as mais diversas práticas locais pois atua como lembrete de etapas simples que favorece o trabalho em equipe e a melhoria da comunicação (Thomé et al., 2021).

\section{Conclusão}

Esta síntese de evidências reúne elementos de verificação para o ambiente cardíaco tendo como base a pergunta de pesquisa do artigo. Confirmações de identificação, nome do procedimento a ser realizado, formulário de consentimento são itens comuns existentes entre as listas encontradas. Os itens específicos para cirurgia cardíaca foram diversos, reunindo informações claras e objetivas quanto a segurança para o paciente cardíaco cirúrgico.

Desse modo, sugere-se o desenvolvimento de estudos com avaliação da efetividade de listas de verificação, quanto à clareza, facilidade do uso, tempo de execução, adesão das equipes e indicadores de melhores desfechos relacionados aos eventos adversos cardíacos cirúrgicos. 


\section{Referências}

Bernardo, T. H., Silva, I, M, F., Lima, S. T., Thomé, A. R. C. S., Soares, I. P., Bernardo, R. C. C., Santos, S. S. G. S. (2021). Identificação dos requisitos necessários para um checklist de cirurgia cardíaca segura. Research Society and Development, 10 (4).

Berrisford, R. G., wilson, I. H., Davidge, M., \& Sanders, S. (2012). Surgical time out checklist with debriefing and multidisciplinary feedback improves venous thromboembolism prophylaxis in thoracic surgery: a prospective audit. Eur j cardiothorac surg, 41(6),1326-1329.

Chan, J., Gupta, A. K., Stewart, S., Babidge, W., Mc Culloch, G., Worthington, M. G., \& Maddern, G. J. (2019). "Nobody told me": Communication Issues Affecting Australian Cardiothoracic Surgery Patients. The Annals of thoracic surgery, 108(6), 1801-1806.

Hussain, S., Adams, C., Cleland, A., Jones, P., Walsh, G., \& Kiaii. B. (2016). Lessons from aviation - the role of checklists in minimally invasive cardiac surgery. Perfusion, 31(1), 68-71.

Joanna Briggs Institute. (2014). Reviewers Manual-Methodology for JBI Mixed Methods Systematic Reviews.

Kim, F. J., da Silva, R. D., Gustafson, D., Nogueira, L., Harlin, T., \& Paul, D. L. (2015). Current issues in patient safety in surgery: a review. Patient Saf Surg, 9 (26).

Konfirst, C., Preston, S., \& Yeh, Jr. T. (2015). Checklists and safety in pediatric cardiac surgery. Semin Thorac Cardiovasc Surg Pediatr Card Surg Annu, $18(1), 43-50$.

Mendes, K. D. S., Silveira, R. C.C. P., \& Galvão, C. M. (2019). Uso de gerenciador de referências bibliográficas na seleção dos estudos primários em revisão integrativa. Texto contexto - enferm, 28:E20170204.

Ogden, S. R., Culp, Jr. W. C., Villamaria, F. J., \& Ball, T. R. (2016). Developing a Checklist: Consensus Via a Modified Delphi. Technique J Cardiothorac Vasc Anesth, 30(4), 855-858.

Organização Mundial da Saúde. (2009). Segundo desafio global para a segurança do paciente: Cirurgias seguras salvam vidas. Rio de Janeiro: Organização Pan-Americana da Saúde/Ministério da Saúde/Agência Nacional de Vigilância Sanitária.

Papaspyros, S. C., Javangula, K. C., \& Adluri, R. K. P. (2010). O'Regan DJ. Briefing and debriefing in the cardiac operating room. Analysis of impact on theatre team attitude and patient safety. Interact Cardiovasc Thorac Surg, 10 (1), 43-47.

Rakoff, D., Akella, K., Guruvegowda, C., Chhajwani, S., Seshadri, S., \& Sola, S. (2018). Improved Compliance and Comprehension of a Surgical Safety Checklist With Customized Versus Standard Training: A Randomized Trial. J Patient Saf, 14 (3), 138-142.

Raman, J., Leveson, N., Samost, A. L., Dobrilovic, N., Oldham, M., Dekker, S., \& Finkelstein., Stan (2016). When a checklist is not enough: How to improve them and what else is needed. J Thorac Cardiovasc Surg, 152 (2), 585-592.

Russ, S., Rout, S., Caris, J., Mansell, J., Davies, R., Mayer, E., Moorthy, K., Darzi, Ara., Vicent, C., \& Sevdalis, N. (2015). Measuring variation in use of the Measuring variation in use of the WHO surgical safety checklist in the operating room: a multicenter prospective cross-sectional study. J Am Coll Surg, 220 (1), 1-11.

Spiess, B. D. (2013). The use of checklists as a method to reduce human error in cardiac operating rooms. Int Anesthesiol Clin, 51(1), 179-194.

Tan, J., Ngwayi, J. R. M., Ding, Z., Zhou, Y., Li, M., Chen, Y., Hu, Bingtao., Liu, Jinping., \& Porter, Daniel. (2021). Attitudes and compliance with the WHO surgical safety checklist: a survey among surgeons and operating room staff in 138 hospitals in China. Patient Saf Surg, 15 (3).

Thomé, A. R. C. S., Melo, E. S., Silva, V. M. S., Almeida, T. G., Farias, I. P. \& Vasconcelos, E. L. (2017). Construção e validação de instrumento para assistência em cirurgia cardíaca segura. Revista de enfermagem UFPE on line.

Thomé, A. R. C. S., Soares, I. P., Bernardo, R. C. C., Santos, S. S. G. S. (2021). Checklist para cirurgia cardíaca segura: revisão integrativa. Research Society and Development 10 (15) e434101523213.

Weiser, T. G., \& Gawande, A. (2015). Excess Surgical Mortality: Strategies for Improving Quality of Care. In H. T. Debas (Eds.) et. al., Essential Surgery: Disease Control Priorities, Third Edition (Volume 1). The International Bank for Reconstruction and Development / The World Bank.

White, M. C., Peven, K., Clancy, O., Okonkwo, I., Bakolis, I., Russ, S., Leather, A., \& Sevdalis, N. (2021). Implementation Strategies and the Uptake of the World Health Organization Surgical Safety Checklist in Low and Middle Income Countries: A Systematic Review and Meta-analysis.Annals of surgery, 273(6), e196-e205.

World Health Organization. (2011). Patient Safety Curriculum Guide Multi-professional Edition. Geneva.

World Health Organization. (2019). World Patient Safety Day. 